\title{
Promonocyte to Lymphocyte Ratio Measurement
}

National Cancer Institute

\section{Source}

National Cancer Institute. Promonocyte to Lymphocyte Ratio Measurement. NCI

Thesaurus. Code C74652.

The determination of the ratio of promonocytes compared to all lymphocytes present in a sample. The measurement may be expressed as a ratio or percentage. 